\title{
Epithelial-to-mesenchymal transition in paired human primary and recurrent glioblastomas
}

\author{
CAROLIN KUBELT $^{1 *}$, KIRSTEN HATTERMANN $^{3 *}$, SUSANNE SEBENS $^{2}$, \\ H. MAXIMILIAN MEHDORN ${ }^{1}$ and JANKA HELD-FEINDT ${ }^{1}$
}

\author{
${ }^{1}$ Department of Neurosurgery, ${ }^{2}$ Institute for Experimental Medicine, Inflammatory Carcinogenesis, \\ University Medical Center Schleswig-Holstein UKSH, Campus Kiel, D-24105 Kiel; \\ ${ }^{3}$ Department of Anatomy, University of Kiel, D-24118 Kiel, Germany
}

Received January 7, 2015; Accepted February 20, 2015

DOI: 10.3892/ijo.2015.2944

\begin{abstract}
Patients with highly malignant glioblastomas have a short median survival time mainly due to aggressive relapses after therapeutic treatment. Beside others, they achieve their progressive character via epithelial-to-mesenchymal transition (EMT). However, comprehensive investigations on EMT in paired primary-recurrent glioblastoma pairs are presently not available. Thus, in our present study we examined the expression profile of different EMT-markers in 17 matched primary and recurrent glioblastomas by qPCR and double-immunofluorescence stainings to identify EMT marker expressing cell types. Additionally, we analyzed the influence of temozolomide on EMT marker expression in vitro. In comparison to primary tumors, expression of $\beta$-catenin $(p<0.05)$, Snaill $(\mathrm{p}<0.05)$, Snail2/Slug $(\mathrm{p}<0.05)$, biglycan $(\mathrm{p}<0.05)$ and Twist1 $(\mathrm{p}<0.01)$ was downregulated in recurrence whereas L1CAM showed upregulation ( $<0.05$; qPCR). Expression of desmoplakin, vimentin, fibronectin and TGF- $\beta 1$ with its receptors TGF- $\beta$ R 1 and TGF- $\beta R 2$ was almost unchanged. Comparing each individual pair, five different 'EMT groups' within our
\end{abstract}

Correspondence to: Professor Janka Held-Feindt, Department of Neurosurgery, University of Schleswig-Holstein Medical Center, Campus Kiel, Arnold-Heller-Str. 3, Building 41, D-24105 Kiel, Germany E-mail: held-feindtj@nch.uni-kiel.de

*Contributed equally

Abbreviations: $\mathrm{BGN}$, biglycan; $\mathrm{C}_{\mathrm{T}}$, cycle of threshold; DMEM, Dulbecco's modified Eagle's medium; DSP, desmoplakin; EMT, epithelial-to-mesenchymal transition; FCS, fetal calf serum; GFAP, GAPDH, glycerinaldehyde-3-phosphate-dehydrogenase; glial fibrillary acidic protein; GMT, glial to mesenchymal transition; Iba1, ionized calcium-binding adapter molecule 1; MET, mesenchymal-to-epithelial transition; Pecam, platelet endothelial cell adhesion molecule; qRT-PCR, quantitative reverse transcription polymerase chain reaction; TGF- $\beta$ (R), transforming growth factor- $\beta$ (receptor); vWF, von Willebrand factor protein; WHO, World Health Organization

Key words: glioblastomas, primary, recurrent, epithelial-tomesenchymal transition, mesenchymal-to-epithelial transition, glial-to-mesenchymal transition, temozolomide glioblastoma collective were identified according to the regulation of mRNA expression of GFAP, desmoplakin, Snail1, Snail2, Twist1 and vimentin. Additionally, double-stainings of EMT markers in combination with cell specific markers (glial fibrillary acidic protein, CD11b, von Willebrand factor) revealed that EMT markers were expressed in a complex pattern with all three cellular types as possible sources. Temozolomide treatment significantly induced mRNA expression of nearly all investigated EMT markers in T98G glioma cells. Thus, EMT seems to be involved in glioma progression in a complex way requiring an individualized analysis, and is influenced by commonly used therapeutic options in glioma therapy.

\section{Introduction}

Biochemical alterations leading to the trans-differentiation of immobile polarized epithelial cells to mesenchymal cells, which are characterized by increased motility and invasiveness, are summarized as epithelial-to-mesenchymal transition (EMT) (1-5). Many aggressive tumors seem to undergo EMT, and this process has been described to facilitate tumor cell invasion, progression and metastasis (1-5). However, EMT seems to be transient as tumor cells in distant metastases of many cancers resemble an epithelial rather than a mesenchymal phenotype. Thus, a process named mesenchymal-to-epithelial transition (MET) describes the reverse phenotypic transition of disseminated mesenchymal tumor cells after recruitment and settlement in target organs $(2,6-8)$. Nevertheless, it is still controversially discussed how both EMT and MET are involved in cancer progression and metastasis (4).

For glioblastomas, highly malignant brain tumors with a median survival of $<15$ months despite multimodal therapy (9), EMT seems to be also a relevant process, although pathophysiological properties and progression modalities are diverging from epithelial tumors (10-14). As one example, the majority of glioblastomas do not show intrinsic E-cadherin expression (15), the classical 'E-cadherin to N-cadherin switch' is unlikely to correlate with mesenchymal transition in glioblastomas (16). Therefore, Mahabir et al proposed the term 'glial to mesenchymal transition (GMT)' based on the aspect that 
glial cells are developmentally derived from the neuroepithelial lineage (11).

Essential molecules described in glioma EMT processes are e.g., ZEB-1 ( $\delta$-crystalline enhancer binding factor-1) and ZEB-2, which are critical regulators of TGF- $\beta$ (transforming growth factor- $\beta$ ) signaling and are responsible for disrupting cell-cell contact inhibition $(10,13)$. In this context, TGF- $\beta$ is not only one of the most potent EMT inducers, but also released by glioma cells in large quantities boostering glioma progression by promoting tumor-angiogenesis, invasion, migration and inhibiting immune responses $(17,18)$. Additionally, TGF- $\beta$ can confer chemoresistance in human glioblastomas e.g., by induction of the neural adhesion molecule L1CAM expression (19). Further EMT-promoting factors are the transcription factors Snail1, Snail2/Slug, and Twist with WNT/ $\beta$-catenin, all having shown to favor glioblastoma cell motility and invasiveness (10-13,20-22). A decrease in the expression of desmoplakin, an obligate component of functional desmosomes that anchors intermediate filaments of desmosal plaques, and desmosome components, has been described in EMT $(17,23)$. Moreover, recently a multi-cancer mesenchymal transition gene expression signature has been identified (24) which contains numerous EMT markers, additional to the above named ones also fibronectin, vimentin and biglycan. Low levels of this signature were shown to be associated with prolonged time until recurrence of the glioblastomas $(25,26)$.

Taken together, all these investigations pointed to a pivotal role of EMT in glioma progression. In fact, Mahabir and colleagues recently showed by qPCR, that in paired probes of malignant gliomas of 7 donors the expression of collagen, matrix metalloproteinase- 9 , smooth muscle $\alpha$-actin ( $\alpha$-SMA), CD44, fibronectin, and YKL-40 were elevated in the recurrent glioma samples. Additionally, they demonstrated by immunohistochemistry that in 22 cases of clinically recurrent gliomas the expression of vimentin, $\alpha$-SMA, and CD44 was increased in relation to primary glioma samples (11). Interestingly, they identified Snaill as a master regulator of irradiationinduced GMT resulting in enhanced migration and invasion of glioma cells. These processes were possibly triggered through phosphorylation of GSK-3 $\beta$ and ERK1/2 activation in a TGF- $\beta$-dependent manner (11). However, the group investigated only a small cohort of 7 matched primary versus recurrent glioblastoma samples and took not into account that transition patterns might be a patient's individual more than a general feature. Additionally, the aspect of the reverse transition MET/MGT has not been addressed properly for matched glioblastoma samples, nor the distribution of epithelial, glial and mesenchymal markers within different cell types of solid human glioblastoma tissues. Thus, we investigated the expression of Twist1, Snail1, Snail2/Slug, desmoplakin, biglycan, $\beta$-catenin, L1CAM, fibronectin, vimentin, and TGF- $\beta 1$ with its receptors TGF- $\beta$ R1 and TGF- $\beta$ R2 in 17 matched probes of solid primary and recurrent human glioblastomas by realtime RT-PCR and double-immunofluorescence staining to identify the individual EMT molecule-expressing cell types. Additionally, to determine the influence of temozomolide, the main chemotherapeutic drug in glioblastoma treatment, on EMT molecules, we stimulated glioma cells with temozolomide and analyzed the expression profile of the above named EMT markers.

\section{Materials and methods}

Tumor specimens. Glioblastoma samples were surgical dissected tissues from the Department of Neurosurgery (Kiel, Germany) and were obtained in accordance with the Helsinki Declaration of 1975 with approval of the ethics committee of the University of Kiel, Germany after written informed consent of donors. Tumors were classified according to the WHO criteria, and the diagnosis was established by a pathologist. In this study, a total number of 34 glioblastomas (17 primary and 17 recurrent tumors, paired samples for each donor) was included. In case of enough material available, matched probes of individual tumor samples were used for different investigations.

Reverse transcription and Real-time PCR (qRT-PCR). RNA was isolated with the TRIzol reagent (Invitrogen, Carlsbad, CA, USA), digested by DNase (Promega, Madison, WI, USA), cDNA was synthesized using RevertAid ${ }^{\mathrm{TM}} \mathrm{H}$ Minus Reverse Transcriptase (Thermo Scientific, Schwerte, Germany) and quantitative reverse transcription real-time PCR (qRT-PCR) was performed as described before $(27,28)$ using TaqMan primer probes (Applied Biosystems, Foster City, CA, USA): $\beta$-catenin (Hs-00172016_m1), biglycan (BGN) (Hs-00156076_m1), desmoplakin (DSP) (Hs-00950591_m1), fibronectin 1 (Hs-00277509_m1), glial acidic fibrillary protein (GFAP) (Hs-00157674_m1), glycerinaldehyde-3-phosphatedehydrogenase (GAPDH) (Hs99999905_m1), ionized calcium-binding adapter molecule 1 (Iba1) (Hs-00610419_g1), L1CAM (Hs-00240928_m1), platelet endothelial cell adhesion molecule (Pecam) (Hs-00169777_m1), Snail1 (Hs-00195591_m1), Sail2/Slug (Hs-00950344_m1), TGF- $\beta$ (Hs-00171257_m1), TGF- $\beta$ R1 (Hs-00610319_m1), TGF- $\beta$ R2 (Hs-00234253_m1), Twist1 (Hs-01675818_s1), vimentin (Hs-00185584_m1). All 34 different primary and recurrent glioblastoma samples as well as temozolomide treated T98G glioma cells were analyzed by qRT-PCR. The reaction was carried out with the MyiQ ${ }^{\mathrm{TM}}$ Single Color Real-time PCR Detection system (Bio-Rad, München, Germany) and fluorescent data were converted into cycle threshold (CT) measurements. $\Delta \mathrm{C}_{\mathrm{T}}$ values of each sample were calculated as $\mathrm{CT}_{\text {gene of interest }}-\mathrm{CT}_{\mathrm{GAPDH}}$. Relative gene expression was calculated with $2^{\text {(normalized CT non-stimulated - normalized CT stimulated) }}=\mathrm{n}$-fold of control. A $\Delta \mathrm{C}_{\mathrm{T}}$ value of 3.33 corresponds to one magnitude lower gene expression compared to GAPDH. For each gene, logarithmic linear dependence of CT-values from the number of copies was verified by using different amounts of cDNA.

To visualize possible similarities according to EMT expression markers, relative gene expression data of individual primary-recurrent glioblastoma pairs were assigned to grey shades. A relative gene expression value of 1 (= equal expression in primary and recurrent glioblastomas) was assigned as $30 \%$ grey, lower $n$-fold expression values (lower expression in recurrent compared to primary) were displayed with increasing lighter shading with 0 corresponding to white. Relative expression values $>1$ (higher expression in recurrence compared to primary) were assigned with increasing darker grey shades until 3-fold induction which was assigned as maximum (black). Afterwards, a 'heatmap-like' arrangement of individual primary-recurrent glioblastoma pairs was 
performed orientating particularly to n-fold values of GFAP, desmoplakin, Snail1, Snail2, Twist1 and vimentin.

Cell culture. The human glioblastoma cell line T98G was obtained from the DKFZ, Heidelberg, Germany, and cultured as reported in Dulbecco's modified Eagle's medium (DMEM; Invitrogen) plus $10 \%$ fetal calf serum (FCS; Invitrogen) as described (29,30). T98G cells were checked for purity by immunostaining with cell type-specific markers and for the absence of Mycoplasma contamination by staining with bisbenzimide as described (29,30).

For stimulation experiments T98G cells were treated with/without temozolomide (Sigma-Aldrich, Taufkirchen, Germany) at $400 \mu \mathrm{g} / \mathrm{ml}$ (adjusted to stock concentrations of $20 \mathrm{mg} / \mathrm{ml}$ in dimethylsulfoxide (DMSO; Sigma-Aldrich) or equal volumes DMSO alone (solvent control) for 24 and $48 \mathrm{~h}$ in DMEM plus $0.5 \%$ FCS, respectively, and afterwards RNA isolation and qPCR were performed as described above.

Immunofluorescence. Cryostat sections of different primary and recurrent glioblastoma tissues were fixed in acetone/methanol $(1: 1 ; 10 \mathrm{~min})$ at $-20^{\circ} \mathrm{C}$, washed with Tris-buffered saline plus $0.1 \%$ Tween-20 (3X TBS-T, room temperature), incubated in $20 \%$, then $70 \%$ ethanol (each 2 min), blocked with Sudan black (1\% in $70 \%$ ethanol) for $10 \mathrm{~min}$, rinsed with $70 \%$ ethanol until dye free, then for 2 min with $20 \%$ ethanol, washed with TBS-T (3X), blocked with $0.1 \%$ bovine serum albumin (PAA Laboratories $\mathrm{GmbH}$, Cölbe, Germany) and $0.2 \%$ glycine in TBS (1 h), then without washing incubated with primary antibodies in TBS-T at $4^{\circ} \mathrm{C}$ overnight (with exception of bovine serum albumin all reagents were purchased from Roth Karlsruhe, Germany). Primary antibodies were omitted for negative controls. After a washing step (3X TBS-T, $10 \mathrm{~min}$ ) the first secondary antibody was incubated for $1 \mathrm{~h}$ at $37^{\circ} \mathrm{C}$ in the dark. The sections were washed with TBS-T (3x10 min). Since all primary antibodies were obtained from mouse, an additional blocking step with donkey anti-mouse $\mathrm{F}(\mathrm{ab})_{2}$-fragments (1:1,000 $1 \mathrm{~h}$, room temperature, from Dianova, Hamburg, Germany) was performed. The second primary antibody was applied after another washing step (3X TBS-T) and incubated overnight at $4^{\circ} \mathrm{C}$. Second primary antibodies were omitted for negative controls. The slides were washed again (3X TBS-T) and incubated with the second secondary antibody for $1 \mathrm{~h}$ at $37^{\circ} \mathrm{C}$. After washing with TBS-T (1×10 min), TBS (2x10 min), nuclei were stained with 4', 6-diamidino-2-phenylindole (DAPI; Invitrogen; 1:30,000, $30 \mathrm{~min}$, room temperature), washed with TBS (3X) and finally distilled water. After embedding in Immu-Mount (Shandon, Pittsburgh, PA, USA) digital photography was performed using a Zeiss microscope and Zeiss camera (Zeiss, Oberkochem, Germany).

In combination with anti-GFAP (1:500, MAB3402, mouse monoclonal, Millipore, Darmstadt, Germany), anti-von Willebrand factor protein (vWF) (1:1,000, sc-53465, mouse monoclonal, Santa Cruz Biotechnology, CA, USA), and anti-CD11b (1:100, sc-1186, mouse monoclonal, Santa Cruz), the specific EMT antibodies anti-Twist1 (1:500, H00007291-M03, mouse monoclonal, Abnova, Heidelberg, Germany); $\beta$-catenin (1:150, 610153, mouse monoclonal; BD Transduction Laboratories, Franklin Lakes, NJ, USA); DSP (undiluted, 651109, mouse monoclonal, Progen Biotechnology
GmbH, Heidelberg, Germany), vimentin (1:50, sc-6260, mouse monoclonal, Santa Cruz), and L1CAM [1:100, L1-11A (subclone UJ127.11); (31) mouse monoclonal], were always stained first with Alexa Fluor 488 coupled secondary antibodies (green, 1:1,000, donkey anti-mouse IgG, Invitrogen), the second secondary antibody detecting the cellular markers was donkey anti-mouse IgG Alexa Fluor 555 (red, 1:1,000, Invitrogen).

Statistical analysis. For statistical analyses the two-tailed Student's t-test with dependent samples was used. Significance levels ranged between ${ }^{*} \mathrm{p}<0.05,{ }^{* *} \mathrm{p}<0.01$ and ${ }^{* * *} \mathrm{p}<0.001$.

\section{Results}

mRNA expression of EMT markers in matched human primary and recurrent glioblastomas. To evaluate mRNA expression levels of different EMT markers in matched probes of human primary and recurrent glioblastomas qRT-PCR analysis was performed. Results are shown in Fig. 1, single $\Delta C_{T}$ values for primary glioblastoma samples are demonstrated in white circles and $\Delta \mathrm{C}_{\mathrm{T}}$ values for recurrent ones in grey triangles. It should be kept in mind that a $\Delta \mathrm{C}_{\mathrm{T}}$ value of 3.33 corresponds to one magnitude lower gene expression. Black filled symbols identify tumor samples which were also used for doubleimmunofluorescence staining.

Irrespective of the tumor source (primary versus recurrent), EMT markers could be detected at considerable levels in glioblastoma tissues. Highest mRNA expression was found for vimentin, fibronectin and TGF- $\beta 1$, respectively, and $\beta$-catenin, TGF- $\beta$ R 2 and BGN showed also prominent mRNA expression levels. In contrast, TGF- $\beta$ R1, Sail2/Slug and DSP, respectively, were found at lower expression levels, and Twist1, Snail1 and L1CAM, respectively, were measurable at lowest levels, although a clear detection of all these markers was possible. The normalized averaged $\Delta \mathrm{C}_{\mathrm{T}}$ values for investigated primary/ recurrent glioblastoma samples were: -0.70/-0.22 (vimentin), 2.68/2.59 (fibronectin), 3.64/3.94 (TGF- $\beta 1$ ), 5.49/6.23 ( $\beta$-catenin), 4.98/5.45 (TGF- $\beta$ R2), 5.82/6.64 (BGN), 9.01/8.98 (TGF- $\beta$ R1), 8.11, 9.22 (Snail2/Slug), 8.96/9.20 (DSP), 9.97/11.63 (Twist1), 10.66/11.62 (Snail1) and 10.35/8.57 (L1CAM), respectively (Fig. 1).

Moreover, although a wide range between single samples was observed and in some samples no detection of certain EMT markers was possible, significant expression differences of some EMT markers were determined in primary and recurrent glioblastomas. Twist1, Snail1, Snail2/Slug and BGN mRNA expression was significantly lower in recurrent glioblastomas than in primary tumors $(\mathrm{p}<0.05$ for Snail1, Snail2/Slug and BGN, $p<0.01$ for Twist1). In contrast, mRNA of L1CAM was found in higher amounts in recurrent glioblastoma samples $(\mathrm{p}<0.05)$. For all other investigated EMT markers no statistically significant different mRNA expression level between matched pairs of primary and recurrent tumor samples in the presented glioblastoma collective was measurable.

To test whether the cellular composition of the tumors significantly differs between investigated matched pairs of glioblastoma samples accounting for the above mentioned findings, in addition mRNA expression levels of GFAP 

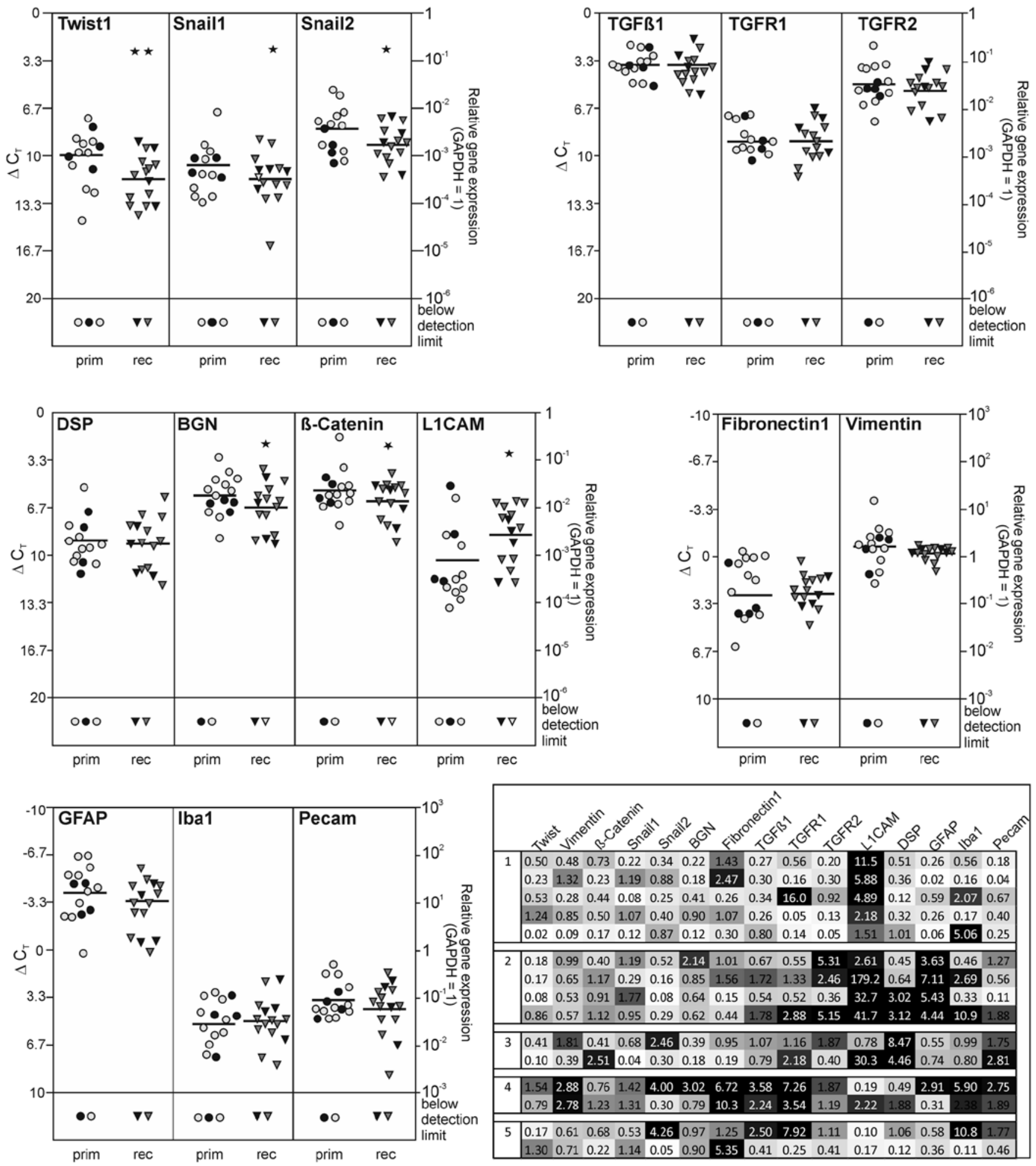

Figure 1. (1) Expression of EMT markers (Twist1, Snail1, Snail2/Slug, desmoplakin (DSP), biglycan (BGN), $\beta$-catenin, L1CAM, fibronectin, vimentin, TGF- $\beta 1$, TGF- $\beta$ R1, TGF- $\beta$ R2) and cellular markers [glial fibrillary acidic protein (GFAP), Ibal and Pecam] in 17 paired primary (prim; white circles) and recurrent (rec; grey triangles) glioblastoma samples was evaluated by real-time RT-PCR (logarithmic scale, $\Delta$ CT $=3.33$ corresponds to a 10 -fold difference; black filled symbols identify samples which were used for immunofluorescence staining); and (2) 'heatmap-like' analysis of n-fold expression differences [calculated with $2^{\text {(normalized CT non-stimulated - normalized CT stimulated) }}=\mathrm{n}$-fold of control) between 15 primary-recurrent glioblastoma pairs [analysis orientated particularly to $\mathrm{n}$-fold values of GFAP, desmoplakin, Snail1, Snail2, Twist1 and vimentin; equal n-fold expression in primary and recurrent pairs was assigned as $30 \%$ grey, lower $n$-fold expression values with increasing lighter shading with 0 corresponding to white, and relative $n$-fold expression values $>1$ with increasing darker grey shades until 3-fold induction which was assigned as maximum (black)]. (1) Twist1, Snaill, Snail2/Slug and BGN mRNA expression in recurrent glioblastoma was significantly lower than for primary one $\left(" \mathrm{p}<0.05 ;{ }^{* *} \mathrm{p}<0.01\right)$, whereas L1CAM was found in higher amounts in recurrent samples ( $\mathrm{p}<0.05)$. (2) In total five different glioblastoma groups could be distiguished, the general de-differentiation group [1], the mesenchymal-epithelial/glial transition group [2], the mesenchymal-epithelial transition group [3], the epithelial-/glial-mesenchymal transition group [4], and the mesenchymal trend group [5]. 
(identifying glial cells), Iba1 (identifying microglia/macrophages) and Pecam (identifying endothelial cells) were also determined. With exception of GFAP, which showed a slightly reduced expression in recurrent glioblastoma samples, no clear expression differences between primary and recurrent glioblastomas concerning these cellular markers were observed.

Since not all of the investigated EMT markers differed between individual primary and recurrent glioblastoma, while for others a wide range of expression amounts were detectable, qRT-PCR results of the individual patients were analyzed in more detail. Therefore, we arranged the $\mathrm{n}$-fold expression changes of 15 individual primary-recurrent glioblastoma pairs in a manner of a heatmap (two pairs which showed constantly no mRNA expression of different EMT markers were omitted for this analysis): equal $\mathrm{n}$-fold expression in primary and recurrent glioblastomas was assigned as $30 \%$ grey, lower $\mathrm{n}$-fold expression values were displayed with increasing lighter shading with 0 corresponding to white, and relative $\mathrm{n}$-fold expression values $>1$ were assigned with increasing darker grey shades until 3-fold induction which was assigned as maximum (black). To identify possible groups of primaryrecurrent glioblastoma pairs according to EMT expression patterns in the values of GFAP, desmoplakin, Snail1, Snail2, Twistl and vimentin were inspected.

Five different glioblastoma groups in our collective were identified: the first group, containing five primary-recurrent glioblastoma pairs, was characterized by a general loss of mRNA expression of all investigated markers, with the exception of L1CAM, which showed higher expression in recurrent glioblastomas. In this group not only GFAP downregulation (designed as our 'glial' marker within a hypothetical glialmesenchymal transition) but also loss of desmoplakin as classical known epithelial marker was found. Since the mesenchymal markers Snail1, Snail2, Twist1 and vimentin were additionally found in lower amounts in recurrent samples, this group was defined as 'general de-differentiation' group. The second group, containing four primary-recurrent glioblastoma pairs, was characterized in addition to L1CAM induction by clearly increased GFAP mRNA expression amounts in recurrent samples, and, moreover, in most cases by higher expression amounts of DSP and the TGF- $\beta$ members, including TGF- $\beta 1$, TGF- $\beta$ R 1 , TGF- $\beta$ R2, and biglycan. In contrast, expression of other mesenchymal markers was mostly reduced in recurrent glioblastomas. Thus, these cases were summarized as "mesenchymal-epithelial/-glial transition' group. The third to fifth group included two glioblastoma pairs, respectively. Whereas the third one was mainly characterized by an increased DSP expression combined with loss of GFAP and inconsistent mesenchymal expression profile in recurrent samples, the 'mesenchymal-epithelial transition' group, the fourth group showed a combination of inconsistent loss of GFAP and DSP with clearly higher mRNA expression amounts of mesenchymal markers in recurrent samples, the 'epithelial-/glial-mesenchymal transition' group. The fifth group was characterized by a clear consistent loss of GFAP and DSP in combination with inconsistent higher expression amounts of mesenchymal markers, the 'mesenchymal trend' group.

Summarized, our results indicate that a broad range of expression profiles of different epithelial, mesenchymal and glial (exemplified for glial acidic fibrillary protein) markers exists in our collective of paired primary and recurrent glioblastoma samples. Various combinations of loss or gain of EMT marker expression are apparently detectable, and that besides screening a whole collective in total, each individual pair has to be analyzed in detail allowing a more precise evaluation of this process underlying the progression from primary to recurrent glioblastoma in individual patients.

Cellular assignment of EMT markers in human primary and recurrent glioblastomas. In order to determine in more detail which cells might account for expression of the EMT markers Twist1, DSP, $\beta$-catenin, L1CAM and vimentin in primary and recurrent glioblastomas, co-stainings with antiglial fibrillary acidic protein (GFAP), anti-von Willebrand factor protein (vWF), and anti-CD11b was performed and analyzed by fluorescence microscopy. The filament protein GFAP identified glial cells, and vWF is a multimeric glycoprotein that is widely used for the detection of endothelial cells in tumor tissues. CD11b, also designated complement component receptor $\alpha 3$ chain or integrin $\alpha \mathrm{M}$, is a cell adhesion molecule that acts as a receptor for cell surface ligands such as intracellular adhesion molecules and is an established marker for microglial/macrophages/monocytes in glioblastoma tissues. Results for primary glioblastomas are shown in Fig. 2, for recurrent ones in Fig. 3.

Since this method does not allow a valid quantification of both staining intensities and amounts of positively stained cells, this analysis exclusively focused on the determination of the cell-specific expression and localisation of EMT markers.

The basic helix-loop-helix transcription factor Twist1 was found to be expressed in GFAP-positive glial cells within both primary and recurrent glioblastoma samples (Figs. 2 and 3, top). Since different markers were not localized within the same structures in the cells, signals did not merge in all cases, but were found in the same regions. Moreover, cells which were positive for either GFAP or Twist1 (or negative for both) existed within the tumor sections. Interestingly, besides a cytoplasmic staining pattern, a nuclear expression of Twist was also observed. Moreover, Twist1 was clearly expressed in vWF-positive endothelial cells and in CD11b-positive microglia/macrophages within primary and recurrent glioblastoma samples. Twistl expression in endothelial cells was clearly more obvious in relation to microglia/macrophages, which showed in some cases only slight Twist1 expression (Figs. 2 and 3, top).

In comparison to Twist1, staining for desmoplakin, an obligate component of functional desmosomes that anchors intermediate filaments of desmosomal plaques, was detectable in lower amounts in primary and recurrent glioblastoma tissues. Nevertheless, expression of desmoplakin could be related to GFAP-positive cell regions, where it was found in clusters as well as in single distributed positive cells within the tissue sections (Figs. 2 and 3; second from top). Since different markers were not localized within the same structures in the cells, signals rarely merged, but were found in the same regions. Whereas vWF-positive cells were also desmoplakin-positive, CD11b-positive cells were almost desmoplakin-negative. As described for Twist1, cells which 


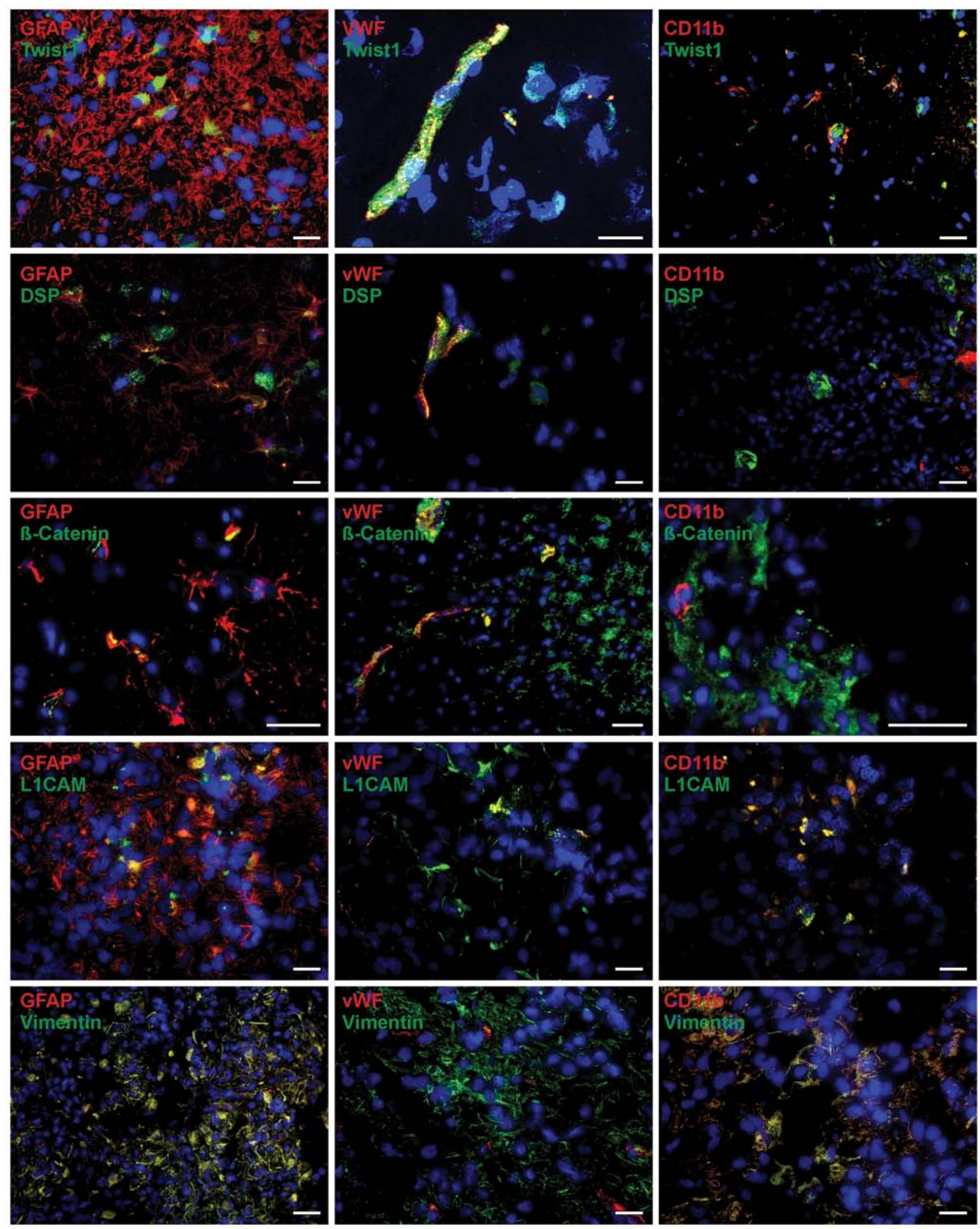

Figure 2. Representative co-staining of EMT markers [Twist1, desmoplakin (DSP), $\beta$-catenin, L1CAM and vimentin] with cellular markers [glial fibrillary acidic protein (GFAP), von Willebrand factor protein (vWF) and CD11b] in primary glioblastoma as determined by immunofluorescence microscopy. EMT markers (green) are found in different combinations with all cellular markers (red) in a complex pattern. Since different markers are not all localized within the same structures in the cells, signals did not merge (yellow) in all cases, but were found in the same regions. Besides double-positive cells also single-positive, either for stained EMT or cellular marker, and double-negative ones were detectable. Magnification, x400; bar, $20 \mu \mathrm{m}$.

were solely positive for GFAP, vWF or desmoplakin (or negative for both) existed within the tumor sections. $\beta$-catenin is a dual function protein, regulating the coordination of cell-cell adhesion and gene transcription, and induces 


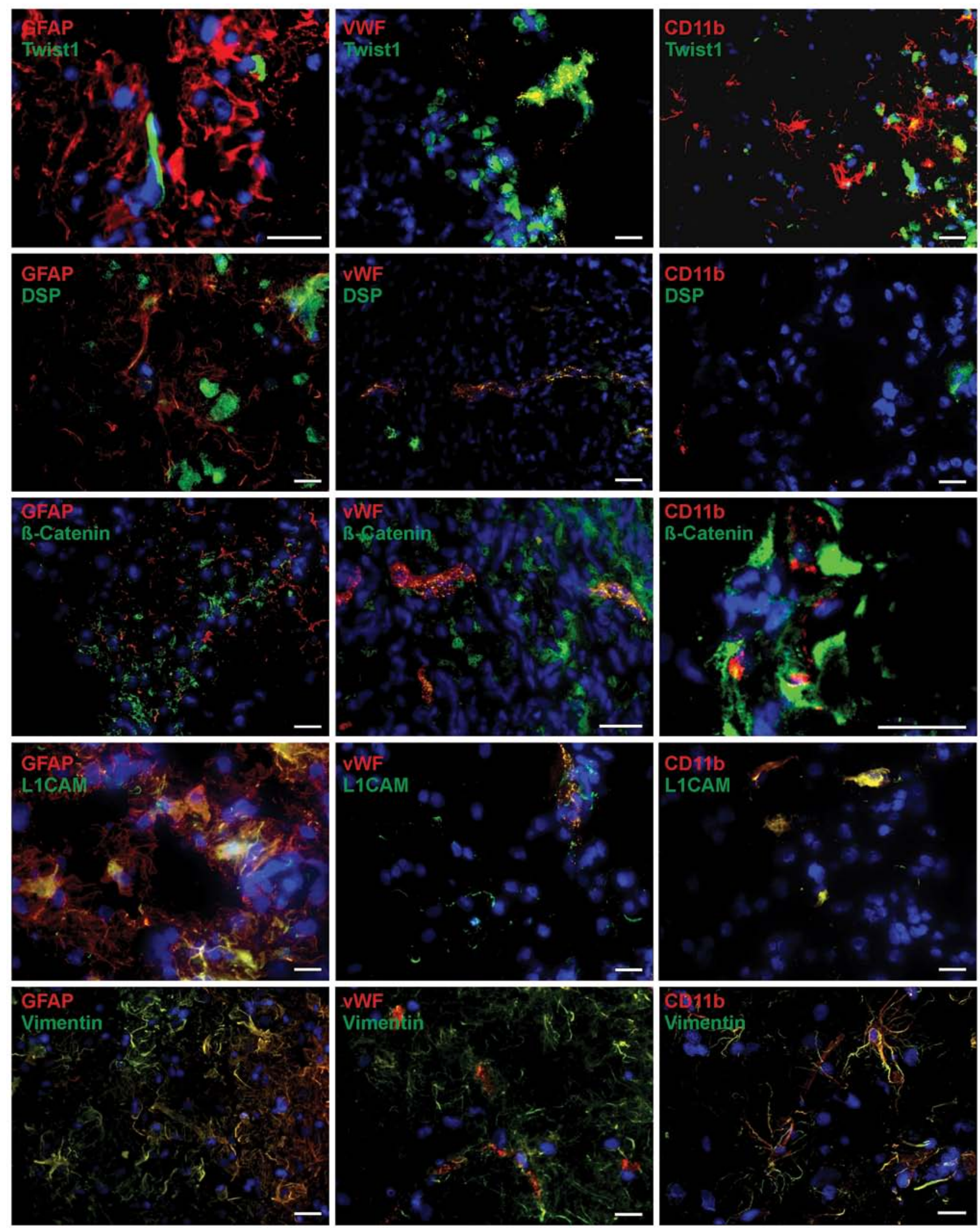

Figure 3. Representative co-staining of EMT markers [Twist1, desmoplakin (DSP), $\beta$-catenin, L1CAM and vimentin] with cellular markers [glial fibrillary acidic protein (GFAP), von Willebrand factor protein (vWF) and CD11b] in recurrent glioblastoma as determined by immunofluorescence microscopy. EMT markers (green) were co-stained with cellular markers (red) in a complex pattern. Concerning the aspect that different markers are not all localized within the same structures in the cells, signals did not merge (yellow) in all cases. Combinations of double-positive, single-positive and double-negative cells were detectable. Magnification, $\mathrm{x} 400$; bar, $20 \mu \mathrm{m}$.

within the WNT/ $\beta$-catenin pathway cell migration which is necessary for pattern formation and differentiation during embryonic development and tumor progression. Staining of $\beta$-catenin in primary and recurrent glioblastomas revealed 

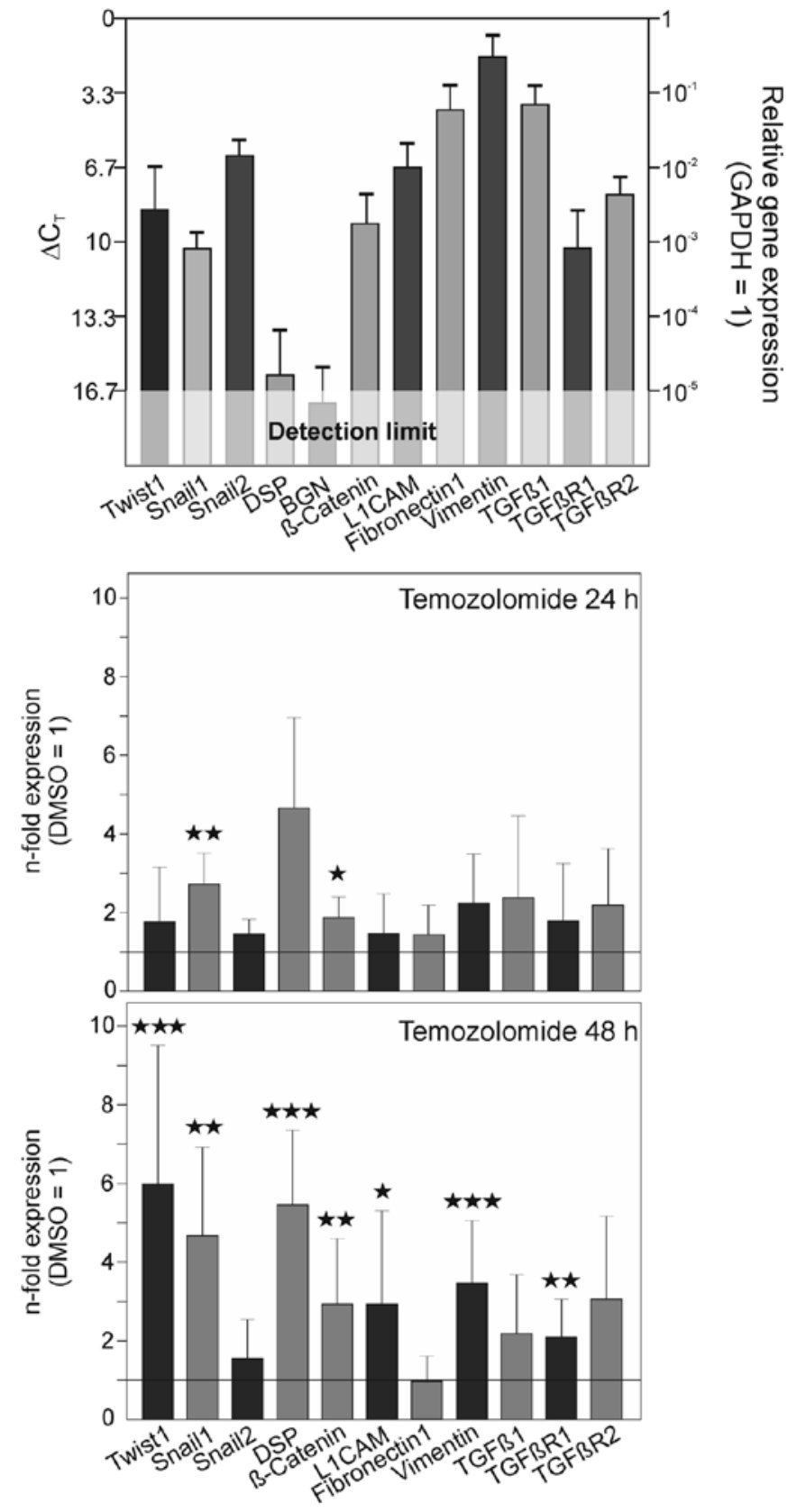

Figure 4. Expression of EMT markers [Twist1, Snail1, Snail2/Slug, desmoplakin (DSP), biglycan (BGN), $\beta$-catenin, L1CAM, fibronectin, vimentin, TGF- $\beta 1$, TGF- $\beta$ R 1 , TGF- $\beta R 2$ ] in untreated (top) and temozolomide-treated (middle, $24 \mathrm{~h}$; bottom, $48 \mathrm{~h}$ ) T98G glioma cells evaluated by real-time RT-PCR (logarithmic scale, $\Delta \mathrm{CT}=3.3$ corresponds to a 10 -fold difference; relative gene expression was calculated with $2^{\text {(normalized CT non-stimulated }}$ - normalized CT stimulated $)=\mathrm{n}$-fold of control; control $=\mathrm{DMSO}=1$ ). With exception of DSP and BGN all investigated EMT markers were expressed in considerable amounts in T98G cells. Temozolomide treatment for up to $48 \mathrm{~h}$ was able to induce mRNA expression of nearly all EMT markers $\left({ }^{*} \mathrm{p}<0.05 ;{ }^{* *} \mathrm{p}<0.01\right.$ $* * * 00.01)$.

$\beta$-catenin-positive cells in GFAP-positive regions, but also, often as clusters of positive cells, near the vWF and CD11bpositive cells (Figs. 2 and 3; third of top). Whereas for endothelial cells a co-staining of vWF and $\beta$-catenin was detectable, CD11b-positive cells seem to be $\beta$-catenin-negative or only slightly positive. In case of GFAP, single glial cells were positive for both GFAP and $\beta$-catenin, whereas several others showed only $\beta$-catenin expression. However, GFAP is a filament protein and $\beta$-catenin has mostly cytoplasmic localization in glioblastoma samples, so these markers have not necessarily to co-localized. Nevertheless, $\beta$-catenin and GFAP were found in the same regions.

The neural adhesion molecule L1CAM (CD171) and the class-III intermediate filament protein vimentin have been associated with EMT along with increased invasion and metastasis in various carcinomas. Indeed, both proteins were expressed in investigated glioblastoma samples revealing robust staining intensities (Figs. 2 and 3). Thus, nearly all GFAP-positive cells were also stained for vimentin, while L1CAM could be localized in GFAP-positive cells but either GFAP-positive or L1CAM-positive cells were detectable, as well. Additionally, it seems that vWF-positive endothelial cells were found to be also positive for either L1CAM or vimentin, those costaining L1CAM with vWF seemed to be more prominent. CD11b-positive cells showed L1CAM and vimentin-positive staining, respectively. As described before, in all tissues the cells solely positive for CD11b GFAP, vWF, L1CAM or vimentin (or negative for both) existed also within the tumor sections.

Summarized, the EMT markers Twist1, DSP, $\beta$-catenin, L1CAM and vimentin were co-stained with GFAP-, vWFand CD11b-positive cells in a complex pattern identifying not only tumor cells but also stromal cells (endothelial cells, macrophages) as possible sources of EMT marker expression. Additionally, no clear expression differences concerning various cell types within solid glioblastoma tissues were detectable between primary and recurrent glioblastoma samples.

Temozolomide treatment induces mRNA expression of different EMT markers. To analyze a possible influence of temozolomide, the most common chemotherapeutic drug in glioblastoma treatment, on the mRNA expression levels of different EMT markers, we stimulated the glioma cell line T98G with temozolomide for 24 and 48 h, respectively. Since this chemotherapeutic drug is dissolved in DMSO equal amounts of this solvent were applied as controls that were used to determine the $\mathrm{n}$-fold induction of mRNA expression as base line values ( $\mathrm{n}$-fold expression for DMSO $=1$ ). Additionally, for determining basal expression levels of all EMT markers, unstimulated T98G cells were investigated. Results are shown in Fig. 4.

The basal expression of all EMT markers was moderate to high, except for DSP and BGN (Fig. 4, top). High mRNA expression was detectable for vimentin, TGF- $\beta 1$ and fibronectin (normalized averaged $\Delta \mathrm{C}_{\mathrm{T}}$ values were $1.75 \pm 0.99$, $3.88 \pm 0.86$ and $4.11 \pm 1$, respectively), moderate expression for Snail2, L1CAM, Twist1 and TGF- $\beta$ R2 (normalized averaged $\Delta \mathrm{C}_{\mathrm{T}}$ values were $6.15 \pm 0.70,6.68 \pm 1.07,8.56 \pm 1.93$ and $7.89 \pm 0.78$, respectively), and lower expression for Snail1, $\beta$-catenin and TGF- $\beta R 1$ (normalized averaged $\Delta C_{T}$ values were $10.31 \pm 0.73,9.19 \pm 1.32$ and $10.29 \pm 1.70$, respectively). Especially for the highly expressed markers (fibronectin, vimentin and TGF- $\beta 1$ ), these results were in accordance with those found in primary and recurrent glioblastoma samples (Fig. 1). Moreover, as described for solid glioblastomas, T98G cells showed a higher expression of TGF- $\beta$ R 2 in relation to 
TGF- $\beta$ R 1 , and Snail2 was more abundantly expressed than Snail1. Only L1CAM and $\beta$-catenin expression was divergent in T98G cells and tumor tissues (L1CAM moderate in T98G versus low in solid samples and vice versa for $\beta$-catenin). Nevertheless, T98G cells can be regarded as a suitable model system to study the effect of temozolomide on EMT marker expression in glioblastoma cells.

For all investigated EMT markers (with exception of BGN which was below detection limit; not shown) an induction of mRNA expression levels after temozolomide treatment was detectable (Fig. 4, middle and bottom). Especially, after 48-h incubation time statistical significant induction of Twist1, DSP, vimentin $(p<0.001)$, Snail1, $\beta$-catenin, TGF- $\beta$ R1 $(\mathrm{p}<0.01)$ and L1CAM $(\mathrm{p}<0.05)$ mRNA was observed. In detail, n-fold induction values were: $5.98 \pm 3.52$ (Twist1), $5.46 \pm 1.99$ (DSP), $3.47 \pm 1.59$ (vimentin), 4.67 \pm 2.26 (Snail1), $2.93 \pm 1.67$ ( $\beta$-catenin), $2.10 \pm 0.95$ (TGF- $\beta R 1)$ and $2.93 \pm 2.39$ (L1CAM). Expression of Snail2, fibronectin, TGF- $\beta 1$ and TGF- $\beta$ R 2 was also induced but without any statistical significance.

Taken together, we were able to show that in paired samples of primary and recurrent glioblastomas a broad range of different EMT markers are expressed in various combinations, and that these markers are expressed in different cell types within solid glioblastoma samples in a complex pattern. Moreover, temozolomide was able to induce expression of nearly all EMT markers, giving a first hint that a chemotherapeutic treatment not only reduces tumor growth by induction of apoptosis, but may also influence the tumor progression in other ways.

\section{Discussion}

EMT is an evolutionary conserved developmental process (32) comprising co-ordinated molecular and cellular changes that result in reduction of cell-cell adhesion, apical-basolateral polarity and epithelial marker expression along with an acquisition of a spindle-cell shape, increased expression of mesenchymal markers and enhanced motility (4). Additionally, EMT is well known to support cancer progression, although its importance is still controversially discussed in the process of cancer metastasis regarding the fact that metastases appear histopathologically similar to the primary tumors from which they are derived (4). Thus, a reversion of this process, called MET, has been postulated which could explain this phenomenon (3). However, experimental data supporting MET in cancer metastasis are still rare mainly due to the limited availability of metastatic tissues (4). Additionally, many invasive and metastatic carcinomas have not undergone complete EMT or invade adjacent connective tissue as individual mesenchymallike cells or even as epithelial cell clusters $(4,33)$. Thus, up to now it is not completely understood which functional role both EMT and MET play in cancer progression and metastasis, particularly applying for glioblastomas.

Malignant gliomas including also their most malignant form, the glioblastomas, frequently occur in the adult brain and represent one of the most aggressive neoplasms in humans $(9,11)$. Despite drastic therapeutic options such as surgical resection, fractionated radiotherapy and adjuvant chemotherapy with alkylating drugs like temozolomide, the incidence of recurrence, regrowth and dissemination of gliomas is still obvious. Thus, several groups are focused on the investigation of EMT processes in gliomas in order to understand in which way this process is involved in glioma progression (10). It was shown that ZEB1 and ZEB2 are responsible for disrupting cell-cell contact inhibition, Twist and Snail family members augment glioblastoma cell motility and invasiveness, and $\beta$-catenin and miR-21 enhance the extracellular matrixcleavage, suggesting that EMT is a major molecular event in glial tumors (10). However, since the majority of glioblastomas do not show intrinsic E-cadherin expression (15), the classical 'E-cadherin to N-cadherin switch' is unlikely to correlate with mesenchymal transition in glioblastomas (16), and therefore the term glial to mesenchymal transition (GMT) was coined (11). Up to now only one group has investigated the EMT process in pairs of primary and recurrent glioblastomas (11). They found by PCR analysis that the expression of collagen, matrix metalloproteinase (MMP)-9, smooth muscle $\alpha$-actin ( $\alpha$-SMA), CD44, fibronectin, and YKL-40 were elevated in the recurrent glioma samples, and demonstrated by immunohistochemistry that in 22 cases of clinically recurrent gliomas the expression of vimentin, $\alpha$-SMA, and CD44 was increased (11). Nevertheless, the group investigated only a small cohort of 7 matched primary and recurrent glioblastoma samples, and did not distinguish in detail which cell types within solid human glioblastomas mainly expressed the EMT markers. Additionally, they did not address the relevance of other processes such as MET or GMT. Thus, we investigated the expression of Twist1, Snail1, Snail2/ Slug, desmoplakin, biglycan, $\beta$-catenin, L1CAM, fibronectin, vimentin, and TGF- $\beta 1$ with its receptors TGF- $\beta$ R1 and TGF- $\beta$ R2 in 17 matched probes of solid primary and recurrent human glioblastomas by real-time RT-PCR as well as by double-immunofluorescence staining to precisely identify EMT molecule-expressing cell types.

We showed that Twist1, Snail1, Snail2/Slug and BGN mRNA expression in recurrent glioblastomas was significantly lower than in primary tumors, whereas mRNA expression of L1CAM was found in higher amounts in recurrent glioblastoma samples. For the other investigated EMT markers no expression differences were detectable. Nevertheless, when investigating each individual pair in detail, a 'heatmap-like' analysis revealed five different groups within our investigated glioblastoma collective. According to the regulation of mRNA expression of mainly GFAP, desmoplakin, Snail1, Snail2, Twist1 and vimentin, we defined a mesenchymal-epithelial/ glial transition group, the 'general-de-differentiation' group, the mesenchymal-epithelial transition group, the epithelial-/ glial-mesenchymal transition group, and the mesenchymal trend group. Additionally, double-immunofluorescence staining revealed that the EMT markers Twist1, DSP, $\beta$-catenin, L1CAM and vimentin could be co-stained with GFAP-, vWFand CD11b-positive cells in a complex pattern identifying all of these three different cellular types as possible sources for EMT marker expression. Thus, our results demonstrate that the EMT, MET and also GMT can all be involved in glioma progression but in a specialized, individual way for each patient's primary-recurrent pair. Since the cellular architecture of glioblastomas is very heterogeneous this result is not really surprising, in particular when considering the aspect that 
the different cell types within glioblastomas, the tumor cells themselves, endothelial cells and microglia/macrophages, are all possible sources of EMT markers. Nevertheless, our results support the view of the existence of distinct 'EMT-phenotypes' in glioma progression which are characterized by congruent expression profiles for certain EMT markers. These results are in accordance with recent investigations of Zarkoob et al (14) showing that the mesenchymal and neural subtypes of glioblastomas have the strongest correlation with a CD133 genetic signature, identifying an aggressive subpopulation of glioblastomas. While the mesenchymal subtype of glioblastomas displays similarity to the signature of both EMT and CD133, it also differs from each of these signatures, possibly due to the fact that signatures of EMT and CD133 are inversely related to each other (14). Additionally, an important role of Snail1 $(11,34)$, Snail2/Slug (25), BGN $(25)$, and Twist1 $(12,35)$ in glioma EMT was previously demonstrated supporting our results, which demonstrated expression differences for these EMT markers between primary and recurrent glioblastomas. Surprisingly, L1CAM, which is well known in glioma progression (19), but was up to now not identified as a classical EMT marker in this tumor entity also showed robust expression differences between paired primary-recurrent samples. The observation that L1CAM expression in gliomas was correlated with high expression of TGF- $\beta$ (36), and that binding of Snail2/Slug has been shown to be essential for TGF- $\beta 1$-induced L1CAM expression in human pancreatic ductal epithelial and pancreatic adenocarcinoma cells (37), point to a similar mechanism underlying L1CAM expression in glioblastomas as well as to its role in EMT in these tumors. In order to substantiate these findings and to clarify the role of EMT and its reversion in glioblastoma progression in more detail, further investigations implying the analysis of a larger cohort of patient-derived samples are certainly required.

Since temozolomide is the main chemotherapeutic drug of glioma therapy, we speculated whether EMT associated processes can be promoted by temozolomide application. To test this, EMT marker expression was analyzed in T89G glioma cells after temozolomide treatment. Indeed, temozolomide was able to induce expression of nearly all EMT markers to considerable amounts in T98G glioma cells. These results are in accordance with the investigations of Mahabir et al (11) showing that especially Snaill was found in higher amounts in glioma cells 21 days after irradiation. Moreover, these cells exhibited an increased migration, invasion and MMP-2 production all these features being abrogated after Snail1 knockdown (11). Altogether these results support the view that different therapeutic options of glioma therapy, including irradiation and chemotherapeutic treatment, are not only beneficial for reducing tumor burden, but may concomitantly favor tumor progression in different ways, e.g., by promoting EMT.

Summarized, EMT/GMT and also MET seemed to be involved in glioma progression in a complex way, the complete understanding requires an individualized analysis. Moreover, these processes seemed to be modulated by certain therapeutic options of glioma therapy potentially antagonizing the expected antitumor effects and providing an explanation for the still high recurrence rate of this tumor entity.

\section{Acknowledgements}

We thank Brigitte Rehmke, Fereshteh Ebrahim and Jörg Krause for expert technical assistance. This study was supported by the University of Kiel and by the popgen 2.0 network [(P2N; supported by a grant from the German Ministry for Education and Research (01EY1103)].

\section{References}

1. Gao D, Vahdat LT, Wong S, Chang JC and Mittal V: Microenvironmental regulation of epithelial-mesenchymal transitions in cancer. Cancer Res 72: 4883-4889, 2012.

2. Scheel C and Weinberg RA: Cancer stem cells and epithelialmesenchymal transition: Concepts and molecular links. Semin Cancer Biol 22: 396-403, 2012.

3. Thiery JP: Epithelial-mesenchymal transitions in tumour progression. Nat Rev Cancer 2: 442-454, 2002

4. Tsuji T, Ibaragi S and Hu GF: Epithelial-mesenchymal transition and cell cooperativity in metastasis. Cancer Res 69: 7135-7139, 2009.

5. Zavadil J, Haley J, Kalluri R, Muthuswamy SK and Thompson E: Epithelial-mesenchymal transition. Cancer Res 68: 9574-9577, 2008.

6. Tarin D, Thompson EW and Newgreen DF: The fallacy of epithelial mesenchymal transition in neoplasia. Cancer Res 65: 5996-6001, 2005.

7. Thompson EW, Newgreen DF and Tarin D: Carcinoma invasion and metastasis: A role for epithelial-mesenchymal transition? Cancer Res 65: 5991-5995, 2005

8. Yates C: Prostate tumor cell plasticity: A consequence of the microenvironment. Adv Exp Med Biol 720: 81-90, 2011.

9. Ohgaki H and Kleihues P: Epidemiology and etiology of gliomas. Acta Neuropathol 109: 93-108, 2005.

10. Kahlert UD, Nikkhah G and Maciaczyk J: Epithelial-tomesenchymal(-like) transition as a relevant molecular event in malignant gliomas. Cancer Lett 331: 131-138, 2013.

11. Mahabir R, Tanino M, Elmansuri A, Wang L, Kimura T, Itoh T, Ohba Y, Nishihara H, Shirato H, Tsuda M and Tanaka S: Sustained elevation of Snail promotes glial-mesenchymal transition after irradiation in malignant glioma. Neuro-oncol 16: 671-685, 2014.

12. Mikheeva SA, Mikheev AM, Petit A, Beyer R, Oxford RG, Khorasani L, Maxwell J-P, Glackin CA, Wakimoto H, González-Herrero I, et al: TWIST1 promotes invasion through mesenchymal change in human glioblastoma. Mol Cancer 9: 194-211, 2010.

13. Pala A, Karpel-Massler G, Kast RE, Wirtz CR and Halatsch M-E: Epidermal to mesenchymal transition and failure of EGFRtargeted therapy in glioblastoma. Cancers (Basel) 4: 523-530, 2012.

14. Zarkoob H, Taube JH, Singh SK, Mani SA and Kohandel M: Investigating the link between molecular subtypes of glioblastoma, epithelial-mesenchymal transition, and CD133 cell surface protein. PLoS One 8: e64169, 2013.

15. Kahlert UD, Maciaczyk D, Doostkam S, Orr BA, Simons B, Bogiel T, Reithmeier T, Prinz M, Schubert J, Niedermann G, et al: Activation of canonical WNT/ $\beta$-catenin signaling enhances in vitro motility of glioblastoma cells by activation of ZEB1 and other activators of epithelial-to-mesenchymal transition. Cancer Lett 325: 42-53, 2012.

16. Brabletz T: To differentiate or not - routes towards metastasis. Nat Rev Cancer 12: 425-436, 2012.

17. $\mathrm{Xu}$ J, Lamouille $\mathrm{S}$ and Derynck R: TGF- $\beta$-induced epithelial to mesenchymal transition. Cell Res 19: 156-172, 2009.

18. Qiao B, Johnson NW and Gao J: Epithelial-mesenchymal transition in oral squamous cell carcinoma triggered by transforming growth factor-beta1 is Snail family-dependent and correlates with matrix metalloproteinase-2 and -9 expressions. Int J Oncol 37: 663-668, 2010

19. Held-Feindt J, Schmelz S, Hattermann K, Mentlein R, Mehdorn HM and Sebens S: The neural adhesion molecule L1CAM confers chemoresistance in human glioblastomas. Neurochem Int 61: 1183-1191, 2012.

20. Elias MC, Tozer KR, Silber JR, Mikheeva S, Deng M, Morrison RS, Manning TC, Silbergeld DL, Glackin CA, Reh TA and Rostomily RC: TWIST is expressed in human gliomas and promotes invasion. Neoplasia 7: 824-837, 2005. 
21. Han SP, Kim JH, Han ME, Sim HE, Kim KS, Yoon S, Baek SY, Kim BS and Oh SO: SNAI1 is involved in the proliferation and migration of glioblastoma cells. Cell Mol Neurobiol 31: 489-496, 2011.

22. Yang HW, Menon LG, Black PM, Carroll RS and Johnson MD: SNAI2/Slug promotes growth and invasion in human gliomas. BMC Cancer 10: 301, 2010

23. De Wever O, Pauwels P, De Craene B, Sabbah M, Emami S, Redeuilh G, Gespach C, Bracke M and Berx G: Molecular and pathological signatures of epithelial-mesenchymal transitions at the cancer invasion front. Histochem Cell Biol 130: 481-494, 2008.

24. Kim H, Watkinson J, Varadan V and Anastassiou D: Multi-cancer computational analysis reveals invasion-associated variant of desmoplastic reaction involving INHBA, THBS2 and COL11A1. BMC Med Genomics 3: 51, 2010.

25. Cheng WY, Kandel JJ, Yamashiro DJ, Canoll $P$ and Anastassiou D: A multi-cancer mesenchymal transition gene expression signature is associated with prolonged time to recurrence in glioblastoma. PLoS One 7: e34705, 2012.

26. Kaufhold S and Bonavida B: Central role of Snail1 in the regulation of EMT and resistance in cancer: A target for therapeutic intervention. J Exp Clin Cancer Res 33: 62, 2014.

27. Tong Y, Mentlein R, Buhl R, Hugo H-H, Krause J, Mehdorn HM and Held-Feindt J: Overexpression of midkine contributes to anti-apoptotic effects in human meningiomas. J Neurochem 100: 1097-1107, 2007.

28. Mentlein R, Forstreuter F, Mehdorn HM and Held-Feindt J: Functional significance of vascular endothelial growth factor receptor expression on human glioma cells. J Neurooncol 67: 9-18, 2004

29. Hattermann K, Held-Feindt J, Lucius R, Müerköster SS, Penfold MET, Schall TJ and Mentlein R: The chemokine receptor CXCR7 is highly expressed in human glioma cells and mediates antiapoptotic effects. Cancer Res 70: 3299-3308, 2010.
30. Held-Feindt J, Hattermann K, Müerköster SS, Wedderkopp H, Knerlich-Lukoschus F, Ungefroren H, Mehdorn HM and Mentlein R: CX3CR1 promotes recruitment of human gliomainfiltrating microglia/macrophages (GIMs). Exp Cell Res 316: 1553-1566, 2010.

31. Mechtersheimer S, Gutwein P, Agmon-Levin N, Stoeck A, Oleszewski M, Riedle S, Postina R, Fahrenholz F, Fogel M, Lemmon $\mathrm{V}$ and Altevogt P: Ectodomain shedding of L1 adhesion molecule promotes cell migration by autocrine binding to integrins. J Cell Biol 155: 661-673, 2001.

32. Thiery JP: Epithelial-mesenchymal transitions in development and pathologies. Curr Opin Cell Biol 15: 740-746, 2003.

33. Friedl $\mathrm{P}$ and Wolf $\mathrm{K}$ : Tumour-cell invasion and migration: Diversity and escape mechanisms. Nat Rev Cancer 3: 362-374, 2003.

34. Myung JK, Choi SA, Kim S-K, Wang K-C and Park S-H: Snail plays an oncogenic role in glioblastoma by promoting epithelial mesenchymal transition. Int J Clin Exp Pathol 7: 1977-1987, 2014.

35. Velpula KK, Dasari VR, Tsung AJ, Dinh DH and Rao JS: Cord blood stem cells revert glioma stem cell EMT by down regulating transcriptional activation of Sox 2 and Twist1. Oncotarget 2: 1028-1042, 2011

36. Tsuzuki T, Izumoto S, Ohnishi T, Hiraga S, Arita N and Hayakawa T: Neural cell adhesion molecule L1 in gliomas: Correlation with TGF-beta and p53. J Clin Pathol 51: 13-17, 1998.

37. Geismann C, Arlt A, Bauer I, Pfeifer M, Schirmer U, Altevogt P, Müerköster SS and Schäfer H: Binding of the transcription factor Slug to the L1CAM promoter is essential for transforming growth factor- $\beta 71$ (TGF- $\beta$ )-induced L1CAM expression in human pancreatic ductal adenocarcinoma cells. Int J Oncol 38: 257-266, 2011 